\title{
ALGORITHMS FOR EMBEDDED MONOIDS AND BASE POINT FREE PROBLEMS
}

\author{
ANNE FAHRNER
}

\begin{abstract}
We present algorithms for basic computations with monoids in finitely generated abelian groups such as monoid membership testing and computing an element of the conductor ideal. Applying them to Mori dream spaces, we obtain algorithms to test whether a Weil divisor class of a given Mori dream space is base point free, to compute generators of the monoid of base point free Cartier divisor classes and to test whether a $\mathbb{Q}$-factorial Mori dream space with known canonical class fulfills Fujita's base point free conjecture or not.
\end{abstract}

\section{INTRODUCTION}

A first part of this paper concerns embedded monoids, that means finitely generated monoids in finitely generated abelian groups, and thereby generalises ideas of the theory on affine semigroups [6. Chapter 2] to monoids with non-trivial torsion part. We further present algorithms for embedded monoids, among others for computing generators of intersections of embedded monoids and for computing an element of the conductor ideal; see Algorithms 2.6-2.12

In the second part of the paper, we apply these algorithms to base point free questions for Mori dream spaces. Recall that Mori dream spaces, introduced by $\mathrm{Hu}$ and Keel 18, are characterized via their optimal behaviour with respect to the minimal model program. A particular interesting aspect of Mori dream spaces is their highly combinatorial structure 2 - in this regard they are a canonical generalisation of toric varieties. Further well-known example classes are spherical varieties [5, smooth Fano varieties 3 and all Calabi-Yau varieties of dimension at most three and with polyhedral effective cone 24. The combinatorial framework developed in 22] allows algorithmic treatment of Mori dream spaces. Applying the aforementioned algorithms to Mori dream spaces, we provide algorithms for testing whether a given Weil divisor class is base point free and for computing generators of the base point free monoid, i.e. the monoid of base point free Cartier divisor classes; see Algorithms 3.3 and 3.4 .

These algorithms, together with the non-emptyness of the conductor ideal of the base point free monoid, play an important role in our main algorithm, Algorithm 4.5, testing Fujita's base point free conjecture [15: this much studied conjecture claims that for a smooth projective variety with canonical class $\mathcal{K}_{X}$, the Weil divisor class $\mathcal{K}_{X}+m \mathcal{L}$ is base point free for all ample Cartier divisor classes $\mathcal{L}$ and for all $m \geq \operatorname{dim}(X)+1$. So far it is known to hold for smooth projective varieties up to dimension five [26, 10, 20, 27]. For toric varieties with arbitrary singularities, Fujino 14 presented a proof of Fujita's base point free conjecture. Despite this substantial progress, Fujita's base point free conjecture remains in general still open. With Algorithm 4.5. we provide a tool for its algorithmic testing for $\mathbb{Q}$-factorial Mori dream spaces. Since our algorithm makes use of the canonical class $\mathcal{K}_{X}$, it applies to Mori dream spaces with known $\mathcal{K}_{X}$. This case appears quite often: for

2010 Mathematics Subject Classification. 14Q15, $20 \mathrm{M} 14$.

Supported by the Carl-Zeiss-Stiftung. 
instance if $X$ is spherical or if its Cox ring is a complete intersection, see Remark 4.2 for details.

In 12, we provide an implementation of our algorithms building on the two Maple-based software packages convex [1] and MDSpackage [17. Using this implementation, we prove Fujita's base point free conjecture for a six-dimensional Mori dream space in Example 4.6 and in Example 4.7. we study a locally factorial Mori dream space that does not fulfill Fujita's base point free conjecture. In addition, we study the more general question of the existence of semiample Cartier divisor classes that are not base point free. It is well-known that for Cartier divisors on complete toric varieties, semiampleness implies base point freeness. For smooth rational projective varieties with a torus action of complexity one and Picard number two, the same statement follows immediately from the classification done in [13. In Example 3.5, we present a first example of a smooth surface of Picard number twelve admitting a semiample Cartier divisor with base points.

The author would like to thank Jürgen Hausen for valuable discussions and comments. In addition, the author is grateful to the referees for their detailed and thorough review of the paper and for their highly appreciated comments and corrections.

\section{EMBEDded Monoids}

Let $K$ be a finitely generated abelian group. We denote by $K=K^{0} \oplus K^{\text {tor }}$ the decomposition of $K$ into free and torsion part and we write $K_{\mathbb{Q}}:=K \otimes_{\mathbb{Z}} \mathbb{Q}$ for the associated rational vector space. Note that each $w \in K=K^{0} \oplus K^{\text {tor }}$ can be represented as $w=\left(w^{0}, w^{\text {tor }}\right)$ with unique elements $w^{0} \in K^{0}$ and $w^{\text {tor }} \in K^{\text {tor }}$. Every $w \in K$ defines an element $w \otimes 1 \in K_{\mathbb{Q}}$, which we denote as well by $w$ for short. A cone in a rational vector space always refers to a convex, polyhedral cone. The relative interior of a cone $\tau \subseteq K_{\mathbb{Q}}$ is denoted by $\tau^{\circ}$.

By an embedded monoid we mean a pair $S \subseteq K$, where $S$ is a finitely generated submonoid of $K$. For an embedded monoid $S \subseteq K$, we denote by

$$
\operatorname{cone}(S):=\operatorname{cone}(w \otimes 1 ; w \in S) \subseteq K_{\mathbb{Q}}
$$

the (convex, polyhedral) cone generated by the elements of $S$. An embedded monoid $S \subseteq K$ is spanning if $S$ generates $K$ as a group. The saturation of an embedded monoid $S \subseteq K$ is the embedded monoid

$$
\tilde{S}:=\left\{w \in K ; n w \in S \text { for some } n \in \mathbb{Z}_{\geq 1}\right\} \subseteq K .
$$

Let $S \subseteq K$ be an embedded monoid. A non-empty set $M \subseteq K$ is called an $S$ module if $S+M \subseteq M$ holds. We call an $S$-module $M$ ideal if $M \subseteq S$ holds and finitely generated if there is a finite subset $\left\{m_{1}, \ldots, m_{\ell}\right\} \subseteq M$ with the property that $M=\left\{m_{1}+s, \ldots, m_{\ell}+s ; s \in S\right\}$ holds.

Definition 2.1. Let $S \subseteq K$ be an embedded monoid. The conductor ideal of $S \subseteq K$ is the set

$$
c(\tilde{S} / S):=\{x \in S ; x+\tilde{S} \subseteq S\} .
$$

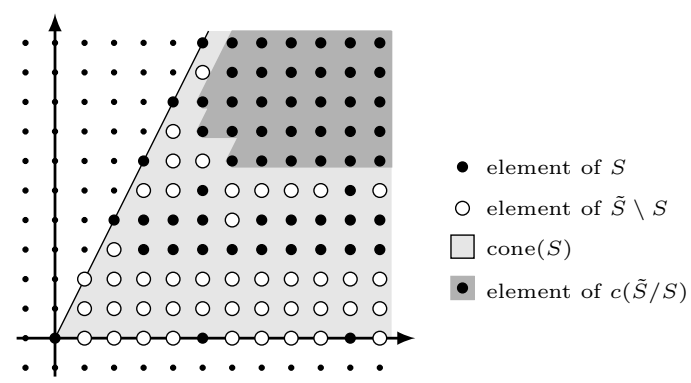


Lemma 2.2. Let $S \subseteq K$ be an embedded monoid. Consider elements $x_{1}, \ldots, x_{r} \in S$ such that $\left\{x_{1} \otimes 1, \ldots, x_{r} \otimes 1\right\}$ is a set of generators for cone $(S)$. Then the finite set

$$
M:=\iota^{-1}\left(\left\{\sum_{i=1}^{r} \alpha_{i}\left(x_{i} \otimes 1\right) ; \quad \alpha_{i} \in \mathbb{Q}, 0 \leq \alpha_{i} \leq 1\right\}\right) \subseteq K,
$$

where $\iota$ is the map $\iota: K \rightarrow K \otimes \mathbb{Q}, w \mapsto w \otimes 1$, generates $\tilde{S}$ as an $S$-module. In particular, $\tilde{S}$ is a finitely generated $S$-module.

Proof. In the case of a torsion free group $K$, the statement on the finite generation of $\tilde{S}$ as an $S$-module is Gordan's Lemma [8, Proposition 1.2.17]. The proof extends easily to the case of finitely generated abelian groups.

Proposition 2.3. Let $S \subseteq K$ be an embedded monoid. If $S \subseteq K$ is spanning, then the conductor ideal $c(\tilde{S} / S)$ is non-empty, i.e. it is in particular an $S$-module.

Proof. By definition, $S+c(\tilde{S} / S) \subseteq c(\tilde{S} / S)$ holds, i.e. we only have to show that $c(\tilde{S} / S)$ is non-empty. In case of a torsion free group $K$, one can find a proof in 6 , Proposition 2.33]. For finitely generated abelian groups we may extend the proof as follows: According to Lemma 2.2. we have $\tilde{S}=\left\{m_{1}+s, \ldots, m_{\ell}+s ; s \in S\right\}$ with some finite subset $\left\{m_{1}, \ldots, m_{\ell}\right\} \subseteq \tilde{S}$. By assumption, the embedded monoid $S \subseteq K$ is spanning. This yields representations $m_{i}=x_{i}-y_{i}$ with $x_{i}, y_{i} \in S$. We claim that $z:=\sum_{i=1}^{\ell} y_{i}$ is contained in the conductor ideal $c(\tilde{S} / S)$. Indeed

$$
z+m_{j}=\sum_{\substack{1 \leq i \leq \ell \\ i \neq j}} y_{i}+x_{j} \in S
$$

holds for all $1 \leq j \leq \ell$, i.e. we have $z+\tilde{S} \subseteq S$.

Lemma 2.4. Let $K$ be a finitely generated abelian group and consider two subgroups $K_{1}, K_{2} \subseteq K$. Let $S_{i} \subseteq K_{i}$ be embedded monoids with saturations $\tilde{S}_{i}$. Then the following holds for the intersection $S_{12}:=S_{1} \cap S_{2}$ :

(i) The intersection $S_{1} \cap S_{2} \subseteq K_{1} \cap K_{2}$ is an embedded monoid.

(ii) We have $\tilde{S}_{12}=\tilde{S}_{1} \cap \tilde{S}_{2}$, where $\tilde{S}_{12}$ denotes the saturation of the embedded monoid $S_{12} \subseteq K_{1} \cap K_{2}$.

(iii) We have $c\left(\tilde{S}_{1} / S_{1}\right) \cap c\left(\tilde{S}_{2} / S_{2}\right) \subseteq c\left(\tilde{S}_{12} / S_{12}\right)$.

Proof. For (i), only the finite generation of $S_{1} \cap S_{2}$ needs some explanation, see for instance [2, Proposition 1.1.2.2]. To prove the first inclusion of (ii), let $x \in \widetilde{S}_{12}$. This means that we have $x \in K_{1} \cap K_{2}$ and that there is $n \in \mathbb{Z}_{\geq 1}$ such that $n x \in S_{1} \cap S_{2}$ holds. Clearly, this shows $x \in \tilde{S}_{1} \cap \tilde{S}_{2}$. To prove the second inclusion, let $x \in \tilde{S}_{1} \cap \tilde{S}_{2}$. Hence $x \in K_{1} \cap K_{2}$ holds and there are $n_{1}, n_{2} \in \mathbb{Z}_{\geq 1}$ such that $n_{i} x \in S_{i}, i=1,2$ hold. This means that $n_{1} n_{2} x$ is contained in $S_{1} \cap S_{2}$, i.e. we have $x \in \tilde{S}_{12}$. For (iii), consider an element $x \in c\left(\tilde{S}_{1} / S_{1}\right) \cap c\left(\tilde{S}_{2} / S_{2}\right)$. This means that $x$ is contained in the intersection $S_{12}$ and that $x+\tilde{S}_{i} \subseteq S_{i}$ holds. With (ii), we conclude that $x+\tilde{S}_{12}$ is contained in $S_{12}$, i.e. the conductor ideal of $S_{12} \subseteq \operatorname{lin}_{\mathbb{Z}}\left(S_{12}\right)$ contains $x$.

Note that the following proposition is not true if we skip the condition that $\operatorname{cone}\left(S_{1}\right)^{\circ}$ and cone $\left(S_{2}\right)^{\circ}$ intersect non-trivially: For instance, $\mathbb{Z}_{\geq 0} \subseteq \mathbb{Z}$ and $\mathbb{Z}_{\leq 0} \subseteq \mathbb{Z}$ define spanning embedded monoids, but the intersection $\{0\} \subseteq \mathbb{Z}$ is not spanning.

Proposition 2.5. Let $K_{1}$ and $K_{2}$ be subgroups of a finitely generated abelian group $K$ and consider embedded monoids $S_{i} \subseteq K_{i}, i=1,2$. If cone $\left(S_{1}\right)^{\circ} \cap \operatorname{cone}\left(S_{2}\right)^{\circ}$ is non-empty and $S_{i} \subseteq K_{i}$ is spanning for $i=1,2$, then $S_{1} \cap S_{2} \subseteq K_{1} \cap K_{2}$ is a spanning embedded monoid. 
Proof. We denote by $S_{12}$ the intersection of $S_{1}$ and $S_{2}$. Note that $S_{12} \subseteq K_{1} \cap K_{2}$ is an embedded monoid by Lemma 2.4 (i). Clearly, the group generated by $S_{12}$ is contained in $K_{1} \cap K_{2}$. It remains to show the opposite inclusion. We denote by $\iota_{1}, \iota_{2}$ and $\iota_{12}$ the maps defined by $w \mapsto w \otimes 1$ fitting into the following diagramm:

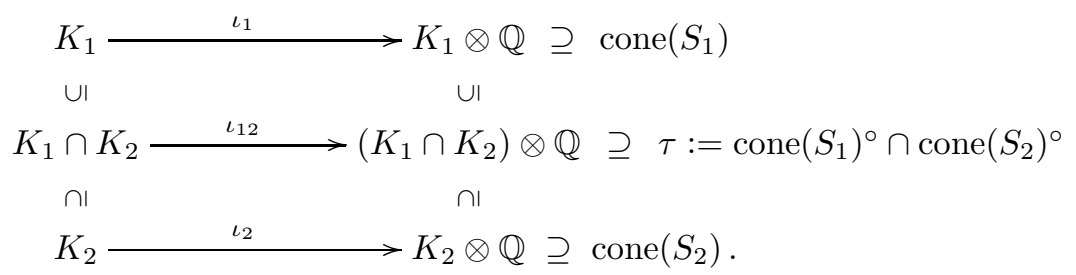

Because of $\tau \neq \emptyset$, the rank of $K_{1} \cap K_{2}$ and the dimension of $\tau$ coincide. Thus there are elements

$$
b_{1}, \ldots, b_{r} \in \iota_{12}^{-1}(\tau) \subseteq \iota_{1}^{-1}\left(\operatorname{cone}\left(S_{1}\right)\right) \cap \iota_{2}^{-1}\left(\operatorname{cone}\left(S_{2}\right)\right)=\tilde{S}_{1} \cap \tilde{S}_{2}
$$

generating $K_{1} \cap K_{2}$ as a group. Furthermore $\tau \neq \emptyset$ implies that there is an element $x \in K_{1} \cap K_{2}$ such that $x \otimes 1 \in \tau$ holds. Recall that $S_{i} \subseteq K_{i}$ are spanning monoids and thus Proposition 2.3 shows that their conductor ideals are non-empty. Since $c\left(\tilde{S}_{i} / S_{i}\right)$ contains some shifted copy of $\tilde{S}_{i}$, there are some $m_{i} \in \mathbb{Z}_{\geq 1}, i=1,2$, such that the integer multiple $m_{i} x$ is contained in $c\left(\tilde{S}_{i} / S_{i}\right), i=1,2$. Hence there is some positive integer $m \in \mathbb{Z}_{\geq 1}$ such that $C$ contains the set of generators $\{m x, m x+$ $\left.b_{1}, \ldots, m x+b_{r}\right\}$ for $K_{1} \cap K_{2}$. It follows that

$$
K_{1} \cap K_{2}=\operatorname{lin}_{\mathbb{Z}}(C) \subseteq \operatorname{lin}_{\mathbb{Z}}\left(c\left(\tilde{S}_{12} / S_{12}\right)\right) \subseteq \operatorname{lin}_{\mathbb{Z}}\left(S_{12}\right)
$$

holds, where the inclusion in the middle was shown in Lemma 2.4 (iii) and the inclusion on the right-hand side follows since $c\left(\tilde{S}_{12} / S_{12}\right)$ is non-empty by the same Lemma and thus contains some shifted copy of $S_{12}$.

In the following we describe some algorithms for monoids which, applied to Mori dream spaces, can be used for computing the base point free monoid $\operatorname{BPF}(X)$, for testing whether a Cartier divisor class is base point free and for computing a point of the conductor ideal of $\operatorname{BPF}(X) \subseteq \operatorname{Pic}(X)$.

Algorithm 2.6 (inMonoid). Input: A finitely generated abelian group $K^{\prime}$, generators $s_{1}^{\prime}, \ldots, s_{t^{\prime}}^{\prime} \in K^{\prime}$ of an embedded monoid $S^{\prime}:=\operatorname{lin}_{\mathbb{Z}_{\geq 0}}\left(s_{1}^{\prime}, \ldots, s_{t^{\prime}}^{\prime}\right) \subseteq K^{\prime}$ and an element $w^{\prime} \in K^{\prime}$.

Output: True if $w^{\prime}$ is contained in $S^{\prime}$. Otherwise, false is returned.

- By excluding the generators $s_{i}^{\prime}$ that equal $0_{K}$, we achieve a representation $S^{\prime}:=\operatorname{lin}_{\mathbb{Z}>0}\left(s_{1}^{\prime}, \ldots, s_{t}^{\prime}\right)$ with a natural number $t \in \mathbb{Z}_{\leq t^{\prime}}$ and with non-zero elements $s_{i}^{\prime}$.

- We compute a canonical representation of the embedded monoid $S^{\prime} \subseteq K^{\prime}$ :

- Compute $r, \tilde{r} \in \mathbb{Z}_{\geq 0}$ such that there is an isomorphism of groups $\varphi: K^{\prime} \rightarrow K:=\mathbb{Z}^{r} \oplus \bigoplus_{k=1}^{\tilde{r}} \mathbb{Z} / a_{i} \mathbb{Z}$.

- Let $S:=\operatorname{lin}_{\mathbb{Z}_{>0}}\left(s_{1}, \ldots, s_{t}\right) \subseteq K$, where we set $s_{i}:=\varphi\left(s_{i}^{\prime}\right) \in K$.

- Set $w:=\varphi\left(w^{\prime}\right) \in K$.

- Let $Q: \mathbb{Z}^{t} \rightarrow K$ denote the homomorphism mapping $x=\left(x_{1}, \ldots, x_{t}\right) \in \mathbb{Z}^{t}$ to the integer combination $\sum x_{i} s_{i}$. Denote by $Q^{0}$ the free part of $Q$, i.e. with the projection $\pi: K \rightarrow K^{0}=K / K^{\text {tor }}$, we have $\pi \circ Q=Q^{0}$.

- Compute the polyhedron $\mathcal{B}:=\left(Q^{0}\right)^{-1}\left(w^{0}\right) \cap \mathbb{Q}_{\geq 0}^{t}$.

- If $\mathcal{B}$ is not bounded, then

- for all $1 \leq i \leq t$ do

$*$ if $s_{i}^{0}=0_{K^{0}}$ holds, then let $\mathcal{C}:=\left\{1 \leq k \leq \tilde{r} ; s_{i r+k} \neq 0\right\}$ and

$$
\mathcal{B}:=\mathcal{B} \cap\left\{x \in \mathbb{Q}^{t} ; x_{i} \leq \prod_{k \in \mathcal{C}} a_{k}\right\} .
$$


- Compute the lattice points of the polytope $\mathcal{B}$, i.e. compute $B:=\mathcal{B} \cap \mathbb{Z}^{t}$.

- Return true if there is a point $x \in B$ such that $Q(x)=w$ holds. Otherwise, return false.

Proof. We first show that in the end of the above algorithm, the polyhedron $\mathcal{B}$ is a polytope. Note that $s_{i} \in K$ is a tupel $s_{i}=\left(s_{i 1}, \ldots, s_{i r}, s_{i r+1}, \ldots, s_{i r+\tilde{r}}\right)$ with integers $s_{i j} \in \mathbb{Z}, 1 \leq j \leq r$, and elements $s_{i r+k} \in \mathbb{Z} / a_{k} \mathbb{Z}, 1 \leq k \leq \tilde{r}$. Via an isomorphism of abelian groups $K \rightarrow K$ we may assume that cone $(S)$ is contained in $\mathbb{Q}_{>0}^{r}$, i.e. we have $s_{i 1}, \ldots, s_{i r} \geq 0$ for all $1 \leq i \leq t$. Consider the polyhedron

$$
\mathcal{A}:=\left(Q^{0}\right)^{-1}\left(w^{0}\right) \cap \mathbb{Q}_{\geq 0}^{t} .
$$

Note that $\mathcal{A}$ contains exactly those lattice points $x=\left(x_{1}, \ldots, x_{t}\right) \in \mathbb{Z}_{>0}^{t}$ with the property that

$$
\sum_{i=1}^{t} x_{i}\left(s_{i 1}, \ldots, s_{i r}\right)=\sum_{i=1}^{t} x_{i} s_{i}^{0}=Q^{0}(x)=w^{0}=\left(w_{1}, \ldots, w_{r}\right)
$$

holds. This means that the integer coefficient $x_{i}$ is smaller than $\left\lfloor\frac{w_{j}}{s_{i j}}\right\rfloor$ for all $1 \leq$ $j \leq r$ with $s_{i j} \neq 0$, where $\lfloor\cdot\rfloor$ denotes the floor function. In particular, we have

$$
\mathcal{A} \subseteq\left\{x \in \mathbb{Q}_{\geq 0}^{t} ; \quad x_{i} \leq \min \left(\left\lfloor\frac{w_{j}}{s_{i j}}\right\rfloor ; 1 \leq j \leq r, s_{i j} \neq 0\right)\right\}
$$

for all $1 \leq i \leq t$ such that $s_{i}^{0} \neq 0_{K^{0}}$ holds, i.e. $\mathcal{A}$ is bounded with respect to these coordinate directions $i$. For all other coordinate directions $1 \leq i \leq t$ of $\mathbb{Z}^{t}$, i.e. of those with $s_{i}^{0}=0_{K^{0}}$, the above algorithm computes a bound $b_{i}$, where

$$
b_{i}:=\prod_{k \in \mathcal{C}} a_{k} \in \mathbb{Z}, \quad \mathcal{C}:=\left\{1 \leq k \leq \tilde{r} ; \quad s_{i r+k} \neq 0_{\mathbb{Z} / a_{k} \mathbb{Z}}\right\} .
$$

Note that $\mathcal{C}$ is non-empty since in the first step of the algorithm, we excluded the $s_{i}^{\prime}$ that are zero. We conclude that

$$
\mathcal{B}=\mathcal{A} \cap\left\{x \in \mathbb{Q}^{t} ; x_{i} \leq b_{i} \text { for all } 1 \leq i \leq t \text { with } s_{i}^{0}=0_{K^{0}}\right\}
$$

is indeed a polytope and thus $B=\mathcal{B} \cap \mathbb{Z}^{t}$ is a finite set.

We now explain why the above algorithm has the claimed output. We need to show that $w^{\prime} \in S^{\prime}$ holds if and only if the algorithm returns true. Clearly, $w^{\prime} \in S^{\prime}$ holds if and only if $w$ is contained in $S$. This in turn is the case if and only if there is an elment $x \in \mathcal{A} \cap \mathbb{Z}_{>0}^{t}$ such that $Q(x)=w$ holds. If $\mathcal{A}$ is a polytope, there is nothing to show. If $\mathcal{A}$ is unbounded we showed above that there is an index $1 \leq i \leq t$ such that $s_{i}^{0}=0_{K^{0}}$ holds. It remains to show that the following assertions are equivalent:

(i) There is an element $x \in \mathcal{A} \cap \mathbb{Z}_{>0}^{t}$ such that $Q(x)=w$ holds.

(ii) There is an element $y \in B_{i}:=\mathcal{A} \cap\left\{x \in \mathbb{Z}_{\geq 0}^{t} ; x_{i} \leq b_{i}\right\}$ with $Q(y)=w$.

Since $B_{i} \subseteq \mathcal{A} \cap \mathbb{Z}_{>0}^{t}$ holds, the direction "(ii) $\Rightarrow(\mathrm{i})$ " is obvious. For the other direction, recall that $b_{i}$ is the product of all $a_{k}, 1 \leq k \leq \tilde{r}$, with $s_{i r+k} \neq 0_{\mathbb{Z} / a_{k} \mathbb{Z}}$. Since $s_{i}^{0}=0_{K^{0}}$ holds, we thus obtain $\alpha s_{i}=\alpha^{\prime} s_{i}$ for all integers $\alpha, \alpha^{\prime}$ with $\alpha \equiv$ $\alpha^{\prime}\left(\bmod b_{i}\right)$. This means that it is sufficient to look at coefficient vectors $x \in \mathbb{Z}_{>0}^{t}$ with $x_{i} \leq b_{i}$, i.e. (i) implies (ii). As argued above, this completes the proof.

Example 2.7. Consider the abelian group $K:=\mathbb{Z} \oplus \mathbb{Z} / 4 \mathbb{Z}$, its elements $s_{1}:=(0, \overline{2})$, $s_{2}:=(1, \overline{1}), s_{3}:=(3, \overline{2}), w:=(3, \overline{1})$ and the monoid $S:=\operatorname{lin}_{\mathbb{Z}_{\geq 0}}\left(s_{1}, s_{2}, s_{3}\right)$ depicted in the picture below. Algorithm 2.6 applied to $S$ and to $w$ does the following:

- The map $Q$ is defined by $\mathbb{Z}^{3} \rightarrow K,\left(x_{1}, x_{2}, x_{3}\right) \mapsto\left(x_{2}+3 x_{3}, \alpha\right)$, where we set $\alpha:=\left(\left(2 x_{1}+x_{2}+2 x_{3}\right)+4 \mathbb{Z}\right) \in \mathbb{Z} / 4 \mathbb{Z}$. Its free part $Q^{0}$ is given by $\mathbb{Z}^{3} \rightarrow \mathbb{Z},\left(x_{1}, x_{2}, x_{3}\right) \mapsto x_{2}+3 x_{3}$. 
- The polyhedron $\left(\mathbb{Q}^{0}\right)^{-1}\left(w^{0}\right)$ is given by $\mathbb{Q} \times\{(3-3 \beta, \beta) ; \beta \in \mathbb{Q}\}$. Thus the algorithm starts with the polyhedron

$$
\mathcal{B}=\mathbb{Q}_{\geq 0} \times\{(3-3 \beta, \beta) ; \beta \in \mathbb{Q}, 0 \leq \beta \leq 1\} .
$$

- Since $\mathcal{B}$ is unbounded and $s_{i}^{0}$ is zero if and only $i=1$ holds, the algorithm then computes the polytope

$$
\mathcal{B}:=\mathcal{B} \cap\left\{x \in \mathbb{Q}^{t} ; x_{i} \leq 4\right\} .
$$

Now we have $\mathcal{B}=\{(\alpha, 3-3 \beta, \beta) ; \alpha, \beta \in \mathbb{Q}, 0 \leq \alpha \leq 4,0 \leq \beta \leq 1\}$.

- In a next step, the algorithm computes the lattice points $B$ of $\mathcal{B}$ :

$$
B=\{(\alpha, 3,0),(\alpha, 0,1) ; \alpha \in \mathbb{Z}, 0 \leq \alpha \leq 4\} .
$$

- Since $Q((1,3,0))=1 s_{1}+3 s_{2}+0 s_{3}=w$ holds, the algorithm returns true.

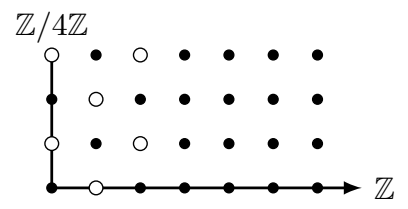

$$
\begin{aligned}
& \text { - element of } S \\
& \text { ○ element of } \tilde{S} \backslash S
\end{aligned}
$$

Algorithm 2.8 (generatorsIntMonoid). Input: Two subgroups $K_{1}, K_{2}$ of a finitely generated abelian group $K$ and generators $s_{i 1}, \ldots, s_{i n_{i}} \in K_{i}$ of embedded monoids $S_{i}:=\operatorname{lin}_{\mathbb{Z}_{>0}}\left(s_{i 1}, \ldots, s_{i n_{i}}\right) \subseteq K_{i}, i=1,2$.

Output: A set of generators for the embedded monoid $S_{1} \cap S_{2} \subseteq K_{1} \cap K_{2}$.

- Let $\varphi:=\varphi_{1} \times \varphi_{2}: \mathbb{Z}^{n_{1}+n_{2}} \rightarrow K \times K$ be the homomorphism of abelian groups defined through $\varphi_{i}: \mathbb{Z}^{n_{i}} \rightarrow K, e_{i j} \mapsto s_{i j}$, where the $e_{i j}$ denote the canonical base vectors of $\mathbb{Z}^{n_{i}}$. Furthermore, define the projection $\psi: K \times K \rightarrow$ $(K \times K) / \Delta$, where $\Delta:=\{(k, k) ; k \in K\}$ denotes the diagonal.

- Compute the kernel of $\beta:=\psi \circ \varphi$.

- Consider the isomorphism of abelian groups $\iota: \mathbb{Z}^{r} \rightarrow \operatorname{ker}(\beta)$ and compute generators $g_{1}, \ldots, g_{t}$ for $\mathbb{Z}^{r} \cap \iota^{-1}\left(\mathbb{Q}_{\geq 0}^{n_{1}+n_{2}}\right)$.

- Define the projection $\pi: K \times K \rightarrow K,(x, y) \mapsto x$ on the first factor and return the set $\left\{(\pi \circ \varphi \circ \iota)\left(g_{j}\right) ; j=1, \ldots, t\right\}$.

Proof. According to Gordan's lemma [8, Proposition 1.2.17], there are generators $g_{1}, \ldots g_{t}$ for the monoid $\mathbb{Z}^{r} \cap \iota^{-1}\left(\mathbb{Q}_{>0}^{n_{1}+n_{2}}\right)$. Let $M:=\operatorname{ker}(\beta) \cap \mathbb{Z}_{>0}^{n_{1}+n_{2}}$ and consider the diagramm

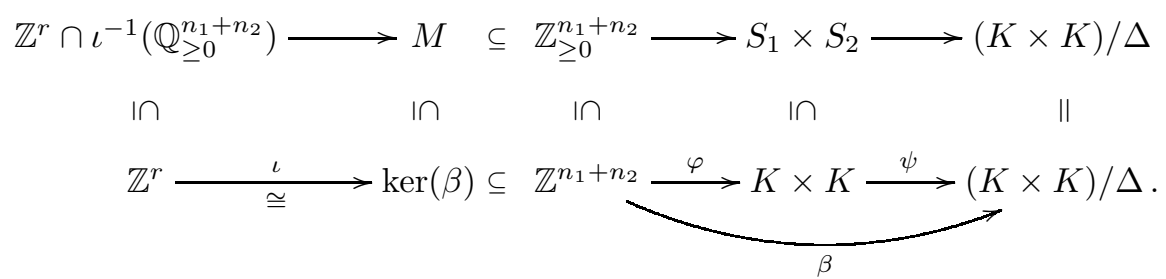

With the projection $\pi: K \times K \rightarrow K,(x, y) \mapsto x$ on the first factor, we obtain

$$
(\pi \circ \varphi \circ \iota)\left(\mathbb{Z}^{r} \cap \iota^{-1}\left(\mathbb{Q}_{\geq 0}^{n_{1}+n_{2}}\right)\right)=(\pi \circ \varphi)(M)=S_{1} \cap S_{2},
$$

where the last equality is true since $\varphi(M)=\left\{(a, b) \in S_{1} \times S_{2} ; a=b\right\}$ holds. We conclude that $\left\{(\pi \circ \varphi \circ \iota)\left(g_{j}\right) ; j=1, \ldots, t\right\}$ is a set of generators for $S_{1} \cap S_{2}$.

Example 2.9. Consider the abelian group $K_{1}:=K_{2}:=K:=\mathbb{Z}$ as well as its elements $s_{11}:=2, s_{12}:=5$, and $s_{21}:=3$. Algorithm 2.8 applied to the monoids $S_{1}:=\operatorname{lin}_{\mathbb{Z} \geq 0}\left(s_{11}, s_{12}\right)$ and $S_{2}:=\operatorname{lin}_{\mathbb{Z}>0}\left(s_{21}\right)$ depicted in the figure below proceeds as follows: 
- The map $\varphi$ is given by $\mathbb{Z}^{3} \rightarrow \mathbb{Z} \times \mathbb{Z}, e_{11} \mapsto s_{11}, e_{12} \mapsto s_{12}, e_{21} \mapsto s_{21}$, where $3=2+1=n_{1}+n_{2}$ holds. To be precise, $\varphi$ is defined by the matrix

$$
\left(\begin{array}{lll}
2 & 5 & 0 \\
0 & 0 & 3
\end{array}\right) \text {. }
$$

- The kernel of $\beta$ is given by $\operatorname{ker}(\beta)=\operatorname{lin}_{\mathbb{Z}}((1,2,4),(0,3,5)) \cong \mathbb{Z}^{2}$.

- The isomorphism $\iota: \mathbb{Z}^{2} \rightarrow \operatorname{ker}(\beta)$ is defined by mapping the first canonical base vector of $\mathbb{Z}^{2}$ to $(1,2,4)$ and the second one to $(0,3,5)$.

- We have $\mathbb{Q}^{2} \cap \iota^{-1}\left(\mathbb{Q}_{\geq 0}^{3}\right)=\operatorname{cone}((3,-2),(0,1))$. According to Gordan's Lemma, computing the lattice points of the polytope

$$
\operatorname{conv}((0,0),(3,-2),(0,1),(3,-1))
$$

gives the following generators for the monoid $\mathbb{Z}^{2} \cap \iota^{-1}\left(\mathbb{Q}_{\geq 0}^{3}\right)$ :

$$
(0,0),(0,1),(1,0),(2,-1),(3,-2),(3,-1) \text {. }
$$

- Applying $\pi \circ \varphi \circ \iota$ to those generators gives the generators $0,15,12,9,6,21$ for $S_{1} \cap S_{2}$. Note that this list is not a hilbert basis. To speed up the computation process in [12, some reduction mechanisms were implemented.
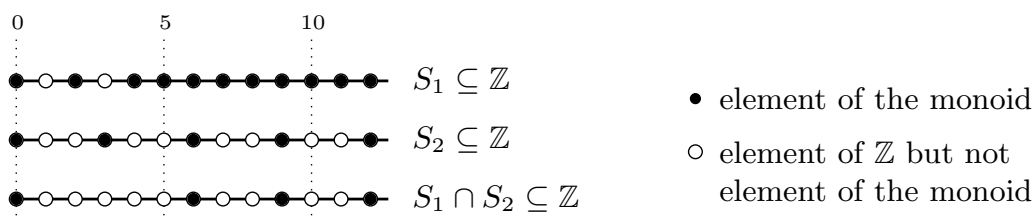

Algorithm 2.10 (inCondIdeal). Input: A finitely generated abelian group $K$, generators $s_{1}, \ldots, s_{t} \in K$ of an embedded monoid $S:=\operatorname{lin}_{\mathbb{Z}_{\geq 0}}\left(s_{1}, \ldots, s_{t}\right) \subseteq K$ and an element $w \in K$.

Output: True if $w$ is contained in $c(\tilde{S} / S)$. Otherwise, false is returned.

- Compute $M$ as defined in Lemma 2.2.

- Use Algorithm 2.6 to test whether $S$ contains $w+M$. Return true if this is the case; otherwise return false.

Proof. Let $w \in K$ and consider $M$ as defined in Lemma 2.2. According to this lemma, $M$ generates $\tilde{S}$ as an $S$-module. This means that the conductor ideal $c(\tilde{S} / S)$ contains $w$ if and only if $w+M$ is contained in $S$.
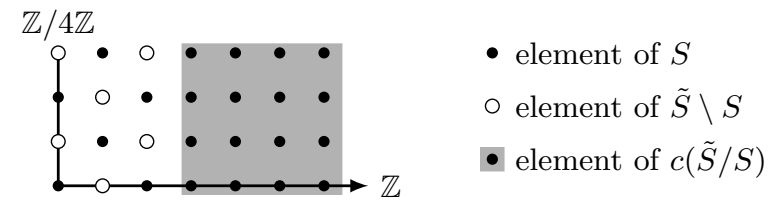

Example 2.11. Consider the abelian group $K:=\mathbb{Z} \oplus \mathbb{Z} / 4 \mathbb{Z}$ as well as its elements $s_{1}:=(0, \overline{2}), s_{2}:=(1, \overline{1}), s_{3}:=(3, \overline{2})$ and the monoid $S:=\operatorname{lin}_{\mathbb{Z}>0}\left(s_{1}, s_{2}, s_{3}\right)$ as in Example 2.7. The monoid and its conductor ideal are illustrated in the above picture. We apply algorithm 2.10 to $w:=(3, \overline{1})$ and test whether $w$ is contained in $c(\tilde{S} / S)$.

- The maps $Q$ and $Q^{0}$ are as in Example 2.7.

- The algorithm computes $M$ as defined in Lemma 2.2. We obtain

$$
M=\{(0, a),(1, a) ; a \in \mathbb{Z} / 4 \mathbb{Z}\} \subseteq K .
$$


- In the next step the algorithm uses Algorithm 2.6 to test whether $S$ contains $w+M=\{(3, a),(4, a) ; a \in \mathbb{Z} / 4 \mathbb{Z}\}$.

- Similarily as in Example 2.7, for $x \in w+M$ with $x^{0}=3$, Algorithm 2.6 computes $B_{3}:=\{(\alpha, 3,0),(\alpha, 0,1) ; 0 \leq \alpha \leq 4, \alpha \in \mathbb{Z}\}$ and we obtain $B_{4}:=\{(\alpha, 4,0),(\alpha, 1,1) ; 0 \leq \alpha \leq 4, \alpha \in \mathbb{Z}\}$ for all $x \in w+M$ with $x^{0}=4$. Since for all $x \in w+M$ with $x^{0}=i, i=3,4$, there is some $y \in B_{i}$ with $Q\left(y_{i}\right)=x_{i}$, the algorithm returns true.

Algorithm 2.12 (pointCondIdeal). Input: A finitely generated abelian group $K$, an element $w \in K$ and generators $s_{1}, \ldots, s_{t} \in K$ of a spanning embedded monoid $S:=\operatorname{lin}_{\mathbb{Z}_{\geq 0}}\left(s_{1}, \ldots, s_{t}\right) \subseteq K$.

Output: A point of the conductor ideal $c(\tilde{S} / S)$.

- Compute $w \in K$ that defines a point in the relative interior of cone $(S)$.

- Use Algorithm 2.10 to compute the smallest integer $r \in \mathbb{Z}_{\geq 1}$ such that $r w$ is contained in $c(\tilde{S} / S)$. Return $r w$.

Proof. This Algorithm terminates since $S \subseteq K$ is spanning.

Example 2.13. Consider the abelian group $K:=\mathbb{Z} \oplus \mathbb{Z} / 4 \mathbb{Z}$ as well as its elements $s_{1}:=(0, \overline{2}), s_{2}:=(1, \overline{1}), s_{3}:=(3, \overline{2})$ and the monoid $S:=\operatorname{lin}_{\mathbb{Z}_{\geq 0}}\left(s_{1}, s_{2}, s_{3}\right)$ as in Examples 2.7 and 2.11 We apply algorithm 2.12 to compute an element of $c(\tilde{S} / S)$.

- At first the algorithm computes the element $(1, \overline{0}) \in K$ defining an element in the relative interior of cone $(S)$.

- For $j=1,2$, Algorithm 2.10 returns that $j(1, \overline{0})$ is not contained in $c(\tilde{S} / S)$.

- In the next step, Algorithm 2.10 shows that $(3, \overline{0})$ is an element of $c(\tilde{S} / S)$.

\section{The base Point Free Monoid of Mori DReam spaces}

We turn to Mori dream spaces and recall the necessary background from 2 . In addition, we present a description of the monoid of base point free Cartier divisor classes of a Mori dream space in terms of combinatorial data, which will be crucial in Algorithm 4.5. We also present algorithms for computing generators of the base point free monoid and for testing whether a Weil divisor class is base point free or not.

Definition 3.1. Let $D$ be a Weil divisor on an irreducible, normal variety $X$ and consider a non-zero section $f \in \Gamma\left(X, \mathcal{O}_{X}(D)\right)$. We call the effective divisor

$$
\operatorname{div}_{D}(f):=\operatorname{div}(f)+D \in \operatorname{WDiv}(X)
$$

the $D$-divisor of $f$. The base locus and the stable base locus of the class $w:=[D] \in$ $\mathrm{Cl}(X)$ are defined as

$$
\operatorname{Bs}(w):=\bigcap_{f \in \Gamma\left(X, \mathcal{O}_{X}(D)\right)} \operatorname{Supp}\left(\operatorname{div}_{D}(f)\right), \quad \mathbf{B}(w):=\bigcap_{n \in \mathbb{Z}_{\geq 0}} \operatorname{Bs}|n D| .
$$

An element $x \in \operatorname{Bs}(w)$ is called a base point of $w$. We call $D \in \operatorname{WDiv}(X)$ or its class $w \in \mathrm{Cl}(X)$ base point free if the base locus $\operatorname{Bs}(w)$ is empty and semiample if its stable base locus is empty. The embedded monoid $\operatorname{BPF}(X) \subseteq \operatorname{Pic}(X)$ of base point free Cartier divisor classes is called base point free monoid of $X$. By $\operatorname{SAmple}(X) \subseteq \mathrm{Cl}(X)_{\mathbb{Q}}$ and $\operatorname{Ample}(X) \subseteq \mathrm{Cl}(X)_{\mathbb{Q}}$, we denote the cones of semiample and ample Weil divisor classes, respectively.

Recall that a Mori dream space is an irreducible normal projective variety $X$ over an algebraic closed field of characteristic zero with finitely generated divisor class group and finitely generated Cox ring

$$
\operatorname{Cox}(X):=\bigoplus_{[D] \in \operatorname{Cl}(X)} \Gamma\left(X, \mathcal{O}_{X}(D)\right)
$$


where in case of torsion in the divisor class group some care is required in this definition, see [2, Section 1.4]. Recall that a non-zero non-unit $f \in \operatorname{Cox}(X)$ is called $\mathrm{Cl}(X)$-prime if it is homogeneous and if $f \mid g h$ with homogeneous elements $g, h \in \operatorname{Cox}(X)$ implies $f \mid h$ or $f \mid g$. Let $\mathfrak{F}:=\left(f_{1}, \ldots, f_{r}\right)$ be a system of pairwise non-associated $\mathrm{Cl}(X)$-prime generators of $\operatorname{Cox}(X)$. Consider the homomorphism of abelian groups $Q: \mathbb{Z}^{r} \rightarrow \mathrm{Cl}(X), e_{i} \mapsto \operatorname{deg}\left(f_{i}\right)$ as well as the total coordinate space $\bar{X}:=\operatorname{Spec}(\operatorname{Cox}(X))$. A face $\gamma_{0} \preceq \gamma$ of the positive orthant $\gamma \subseteq \mathbb{Q}^{r}$ is called an $\mathfrak{F}$-face, if there is a point $x \in \bar{X}$ with $x_{i} \neq 0 \Leftrightarrow e_{i} \in \gamma_{0}$ for all $1 \leq i \leq r$. The collection of relevant faces and the covering collection are

$$
\begin{gathered}
\operatorname{rlv}(X):=\left\{\gamma_{0} \preceq \gamma ; \gamma_{0} \mathfrak{F} \text {-face with Ample }(X)^{\circ} \subseteq Q\left(\gamma_{0}\right)^{\circ}\right\}, \\
\operatorname{cov}(X):=\left\{\gamma_{0} \in \operatorname{rlv}(X) ; \gamma_{0} \text { minimal with respect to inclusion }\right\} .
\end{gathered}
$$

Note that an ample Weil divisor class $u \in \mathrm{Cl}(X)$ together with $\mathfrak{F}$ and relations of $\operatorname{Cox}(X)$ fixes a Mori dream space up to isomorphism: one can reconstruct $X$ as GIT-quotient $p_{X}: \bar{X}^{\mathrm{ss}}(u) \rightarrow X$ of the set of $H$-semistable points $\bar{X}^{\mathrm{ss}}(u) \subseteq \bar{X}$ regarding the action of $H:=\operatorname{Spec} \mathbb{K}(\operatorname{Cl}(X))$ on $\bar{X}$, see for instance [2, Section 3.1].

To any relevant face $\gamma_{0} \preceq \gamma$ we associate as in [2, Construction 3.3.1.1] the set $X\left(\gamma_{0}\right):=p_{X}\left(\bar{X}\left(\gamma_{0}\right)\right)$, where we have

$$
\bar{X}\left(\gamma_{0}\right):=\left\{x \in \bar{X} ; \quad f_{i}(x) \neq 0 \Leftrightarrow e_{i} \in \gamma_{0} \text { for } 1 \leq i \leq r\right\} \subseteq \bar{X}^{\mathrm{ss}}(u) .
$$

According to 2, Corollary 3.3.1.6.] and to [2, Proposition 3.3.2.8], the Picard group $\operatorname{Pic}(X)$ of $X$ and the base locus of an element $w \in \mathrm{Cl}(X)$ are given by

$$
\operatorname{Pic}(X)=\bigcap_{\gamma_{0} \in \operatorname{cov}(X)} Q\left(\operatorname{lin}\left(\gamma_{0}\right) \cap E\right) \quad \text { and } \quad \operatorname{Bs}(w)=\bigcup_{\substack{\gamma_{0} \in \operatorname{rlv}(X) \\ w \notin Q\left(\gamma_{0} \mathbb{Z}^{r}\right)}} X\left(\gamma_{0}\right) .
$$

For projective varieties, any Cartier divisor is the difference of two very ample divisors [9, 1.20]. Thus, the base point free monoid of projective varieties is a spanning embedded monoid. By Proposition 2.3 this means in particular that its conductor ideal is non-empty. For Mori dream spaces, we retrieve the same result in the following Corollary. In addition, we give a description of $\operatorname{BPF}(X)$ in terms of the covering collection and the homomorphism $Q: \mathbb{Z}^{r} \rightarrow \mathrm{Cl}(X), e_{i} \mapsto \operatorname{deg}\left(f_{i}\right)$.

Corollary 3.2. In the above notation, the base point free monoid $\operatorname{BPF}(X) \subseteq$ $\operatorname{Pic}(X)$ of a Mori dream space $X$ is a spanning embedded monoid given by

$$
\operatorname{BPF}(X)=\bigcap_{\gamma_{0} \in \operatorname{cov}(X)} Q\left(\gamma_{0} \cap \mathbb{Z}^{r}\right) .
$$

Proof. The representation of $\operatorname{BPF}(X)$ as an intersection of monoids $Q\left(\gamma_{0} \cap E\right)$ is an immediate consequence of the above description of base loci. In addition, for each $\gamma_{0} \in \operatorname{cov}(X)$, the embedded monoid $Q\left(\gamma_{0} \cap E\right) \subseteq Q\left(\operatorname{lin}\left(\gamma_{0}\right) \cap E\right)$ is spanning. Using the above description of $\operatorname{Pic}(X)$ together with Proposition 2.5. we obtain that $\operatorname{BPF}(Z) \subseteq \operatorname{Pic}(Z)$ is a spanning embedded monoid.

Note that the following algorithms build on the maple-based software package MDSpackage [17. A Mori dream space $X$ is entered and stored in terms of an ample class $u$ together with pairwise non-associated $\mathrm{Cl}(X)$-prime generators and relations of $\operatorname{Cox}(X)$. As explained above, this data fixes a Mori dream space up to isomorphism.

Algorithm 3.3 (generatorsBPF). Input: A Mori dream space.

Output: A set of generators for the embedded monoid $\operatorname{BPF}(X) \subseteq \operatorname{Pic}(X)$.

- Use MDSpackage to compute the covering collection of $X$.

- Use Algorithm 2.8 to compute generators of the intersection

$$
\bigcap_{\gamma_{0} \in \operatorname{cov}(X)} Q\left(\gamma_{0} \cap \mathbb{Z}^{r}\right) \text {. }
$$


Algorithm 3.4 (isBasePointFree). Input: A Mori dream space $X$ and a Weil divisor class $w \in \mathrm{Cl}(X)$.

Output: True if $w$ is base point free. Otherwise, false is returned.

- Use Algorithm 3.3 to compute generators of $\operatorname{BPF}(X) \subseteq \operatorname{Pic}(X)$.

- Apply Algorithm 2.6 to $w$ and $\operatorname{BPF}(X)$.

Using the implementation given in 12, we study the question of the existence of semiample Cartier divisor classes that are not base point free. It is well-known that for Cartier divisors on complete toric varieties, semiampleness implies base point freeness, see for instance [8, Theorem 6.3.12.]. For smooth rational projective varieties with a torus action of complexity one and Picard number two, the same statement follows immediately from the classification done in [13. Note that the discrepancy between semiampleness and base point freeness of divisors on varieties with a torus action of complexity one is already fairly well understood in the language of polyhedral divisors: A criterion for semiampleness is given in [25, Theorem 3.27] and a criterion for base point freeness was proved in [19, Theorem 3.2].

Example 3.5. We give an example of a smooth Mori dream $\mathbb{K}^{*}$-surface that admits semiample Cartier divisor classes with base points.

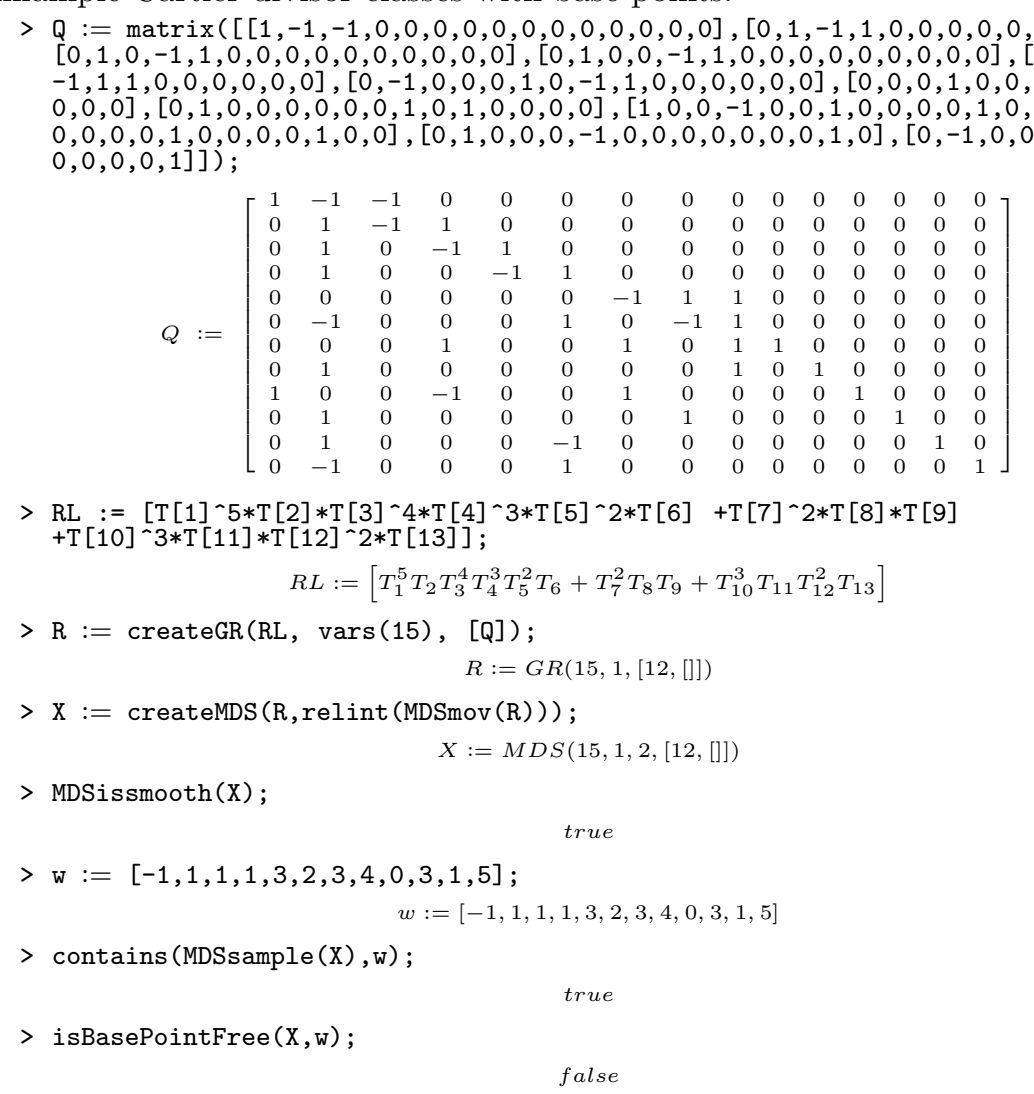

The computation shows that $w=[-1,1,1,1,3,2,3,4,0,3,1,5]$ is a semiample but not base point free Cartier divisor class.

For a geometric interpretation note that $X$ is obtained by blowing up $\mathbb{P}_{1} \times \mathbb{P}_{1}$ ten times in the following way: One considers the $\mathbb{K}^{*}$-action on $\mathbb{P}_{1} \times \mathbb{P}_{1}$ given by

$$
t \cdot\left(\left[y_{0}, y_{1}\right],\left[z_{0}, z_{1}\right]\right):=\left(\left[y_{0}, y_{1}\right],\left[z_{0}, t z_{1}\right]\right) .
$$

The fixed points lie on the two curves $C_{1}:=\mathbb{P}_{1} \times\{[0,1]\}$ and $C_{1}:=\mathbb{P}_{1} \times\{[1,0]\}$. In order two obtain $X$, one blows up the three fixed points $c_{11}:=\{[0,1],[0,1]\} \in C_{1}$, $c_{12}:=\{[1,0],[0,1]\} \in C_{1}$ and $c_{21}:=\{[1,-1],[1,0]\} \in C_{2}$. The resulting hyperbolic fixed points are again blown up: for $c_{11}$, one repeats this four times, for $c_{12}$, one 
repeats this two times and for $c_{21}$ just once. The resulting variety then is isomorphic to $X$.

\section{FUJita BASE POINT FREE TEST}

In the end of the eighties, Takao Fujita conjectured the following:

Conjecture 4.1 (Fujita's base point free conjecture 15). Let $X$ be an n-dimensional smooth projective variety with canonical class $\mathcal{K}_{X}$ and let $\mathcal{L}$ be an ample Cartier divisor class. Then the following holds:

$$
\mathcal{K}_{X}+m \mathcal{L} \text { is base point free for all } m \geq n+1 \text {. }
$$

In order to test whether a $\mathbb{Q}$-factorial Mori dream space $X$ with known canonical class fulfills Fujita's base point free conjecture, we need to test whether $\mathcal{K}_{X}+m \mathcal{L}$ is an element of $\operatorname{BPF}(X)$ for all $m \geq \operatorname{dim}(X)+1$ and for all ample Cartier divisor classes $\mathcal{L}$. Since we can only carry out finitely many tests, we encouter two problems: firstly, we need to bound $m$ and secondly, we need to find a finite validation set of Cartier divisor classes $\mathcal{L}$. In this section, we introduce our solution to these problems and also present some examples of applying our test algorithm.

Remark 4.2. Algorithm 4.5 applies to Mori dream spaces with known canonical class. For instance, if $\operatorname{Cox}(X)$ is a complete intersection, there is a concrete formula for the canonical class in terms of generators and relations of $\operatorname{Cox}(X)$ 2, Proposition 3.3.3.2]. Note that all irreducible normal rational projective varieties with a torus action of complexity one have a complete intersection Cox ring [16. Proposition 1.2]. In addition, there are formulas for the canonical class of spherical varieties, see [4, 22.

Construction 4.3. Let $K^{0}$ be a lattice. Consider an $s$-dimensional cone $\sigma \subseteq K_{\mathbb{Q}}^{0}$ with some facet $F \preceq \sigma$. Let $\varphi: K^{0} \rightarrow \mathbb{Z}^{n}$ be an isomorphism of $\mathbb{Z}$-modules such that $\varphi(\sigma) \subseteq \operatorname{cone}\left(e_{1}, \ldots, e_{s}\right)$ and $\varphi(F) \subseteq \operatorname{cone}\left(e_{1}, \ldots, e_{s-1}\right)$ holds, where $e_{1}, \ldots, e_{n}$ denote the canonical base vectors of the rational vector space $\mathbb{Q}^{n}$. For any $k \in \mathbb{Z}$ we call $\tau:=\varphi^{-1}(\tilde{\tau})$ the $k$-th facet parallel of $F$, where we set

$$
\tilde{\tau}:=\left(\operatorname{lin}_{\mathbb{Q}}(\varphi(F))+k e_{s}\right) .
$$

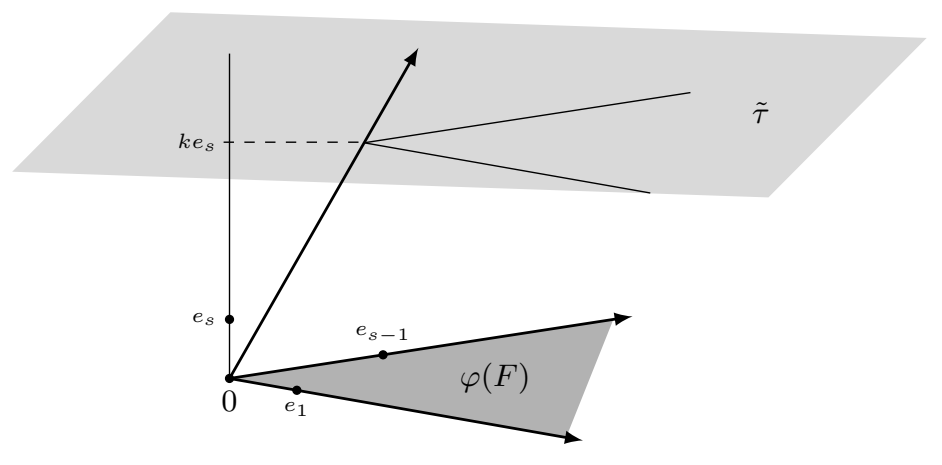

Setting 4.4. Let $X$ be a $\mathbb{Q}$-factorial Gorenstein Mori dream space and consider the base point free monoid $S:=\operatorname{BPF}(X) \subseteq K:=\operatorname{Pic}(X)$. We denote by $F_{1}, \ldots, F_{r}$ the facets of $\sigma:=\operatorname{cone}\left(w^{0} \otimes 1 ; w \in S\right) \subseteq K_{\mathbb{Q}}^{0}$. Let $1 \leq i \leq r$ and let $m_{1}, \ldots, m_{n_{i}} \in S$ be those elements such that $m_{j}^{0}$ is minimal with the property that $m_{j}^{0} \otimes 1$ is contained in a ray of $F_{i}$. Consider the polytope

$$
G_{i}:=\left\{\sum_{j=1}^{n_{i}} a_{j}\left(m_{j}^{0} \otimes 1\right) ; \quad a_{j} \in \mathbb{Q}, 0 \leq a_{j} \leq 1\right\} \subseteq F_{i}
$$


as indicated in the figure below and let $\rho_{1}, \ldots \rho_{t_{i}}$ be the rays of $\sigma$ that are not contained in $F_{i}$. We denote by $\tau_{i}^{k}$ the $\mathrm{k}$-th facet parallel of $F_{i}$. For each facet parallel $\tau_{i}^{k}$ with $k \in \mathbb{Z}_{\geq 0}$, we denote by $p_{j}^{k} \in K_{\mathbb{Q}}, 1 \leq j \leq t_{i}$, the point that is the intersection of $\rho_{j}$ and $\tau_{i}^{k}$. With the canonical embedding $\iota_{0}: K^{0} \rightarrow K_{\mathbb{Q}}^{0}, w \mapsto w \otimes 1$, we define

$$
\begin{aligned}
P_{i}^{k} & :=\left(\operatorname{conv}\left(p_{1}^{k}, \ldots, p_{t_{i}}^{k}\right)+G_{i}\right) \cap \sigma^{\circ} \subseteq \tau_{i}^{k} \text { and } \\
G p_{i}^{k} & :=\iota_{0}^{-1}\left(P_{i}^{k}\right) \times K^{\text {tor }} \subseteq K
\end{aligned}
$$

for all $k \in \mathbb{Z}_{\geq 0}$, where $\sigma^{\circ}$ denotes the relative interior of $\sigma$. Consider the canonical class $\mathcal{K}_{X} \in K$ of $X$. Since $S \subseteq K$ is spanning, there is an element $C \in c(\tilde{S} / S)$. For $1 \leq i \leq r$ let $\alpha_{i} \in \mathbb{Z}$ such that $\left(-\mathcal{K}_{X}^{0}+C^{0}\right) \otimes 1 \in \tau_{i}^{\alpha_{i}}$ holds and set $\nu:=$ $\max \left(\alpha_{i} ; 1 \leq i \leq r\right)$. Note that $\alpha_{i}$ may be negative.

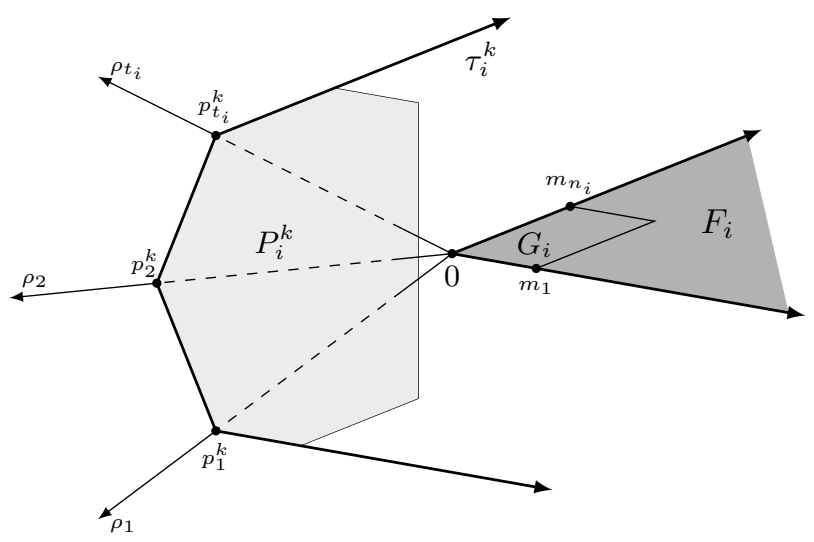

The above mentioned problems, namely bounding $m$ and finding a finite validation set of Cartier divisor classes, are tackled by computing a point of the conductor ideal of $\operatorname{BPF}(X)$ and by only considering the Cartier divisor classes defining a point in the polytopes $P_{i}^{k}$ of the first few facet parallels $\tau_{i}^{k}, k \geq 0$, of each facet $F_{i} \preceq \sigma$.

Algorithm 4.5 (fujitaBpf). Input: A $\mathbb{Q}$-factorial Mori dream space $X$ and its canonical class $\mathcal{K}_{X}$.

Output: True if $X$ fulfills Fujita's base point free conjecture, i.e. if $\mathcal{K}_{X}+m \mathcal{L}$ is base point free for all $m \geq \operatorname{dim}(X)+1$ and all ample Cartier divisor classes $\mathcal{L}$. Otherwise, false is returned.

- If $X$ is not Gorenstein return false.

- Use Algorithm 2.8 to compute generators of $S:=\operatorname{BPF}(X)$.

- Use Algorithm 2.12 to compute a point $C \in c(\tilde{S} / S)$.

- Compute the facets $F_{1}, \ldots, F_{r}$ of cone $(\mathrm{S})$ and $\alpha_{1}, \ldots, \alpha_{r}$ as well as $\nu$ as defined in Setting 4.4 .

- For each $1 \leq i \leq r$ do

- for each $\operatorname{dim}(X)+1 \leq m \leq \nu-1$ do

* for each $1 \leq k \leq\left\lfloor\frac{\alpha_{i}-1}{m}\right\rfloor$, where $\lfloor\cdot\rfloor$ denotes the floor function, use Algorithm 2.6 to test whether $\mathcal{K}_{X}+m G p_{i}^{k} \subseteq S$ holds.

- Return false if there is $1 \leq i \leq r, \operatorname{dim}(X)+1 \leq m \leq \nu-1,1 \leq k \leq\left\lfloor\frac{\alpha_{i}-1}{m}\right\rfloor$, and $\mathcal{L} \in G p_{i}^{k}$ such that $\mathcal{K}_{X}+m \mathcal{L}$ is not contained in $S$. Otherwise, return true.

Before presenting a proof of Algorithm 4.5 we first give two examples of applying it to Mori dream spaces.

Example 4.6. Here we give an example of a six-dimensional smooth Mori dream space that does fulfill Fujita's base point free conjecture. 


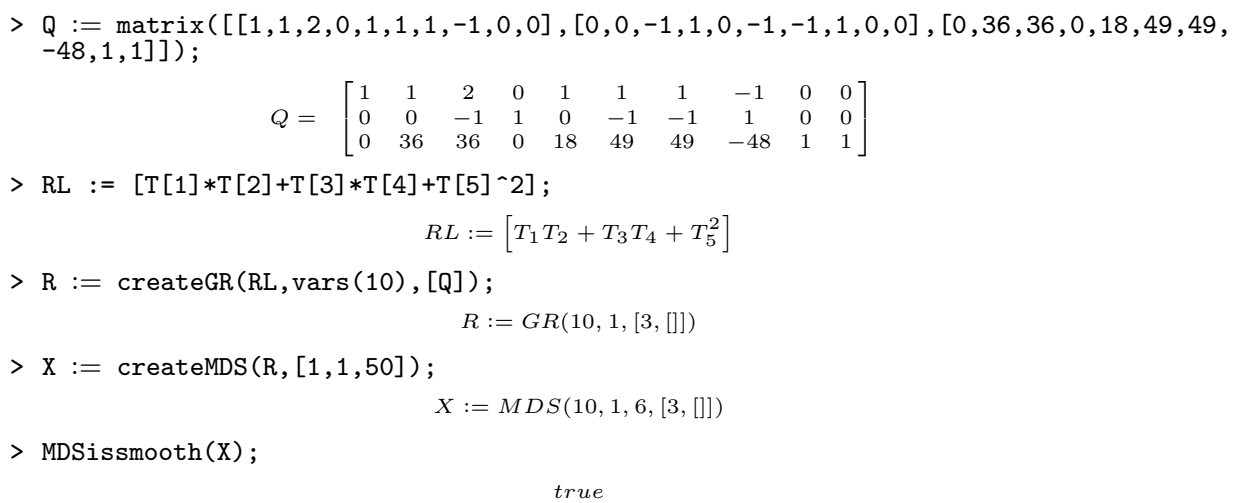

Since $R=\operatorname{Cox}(X)$ is a complete intersection, we may use the formula presented in 2 to compute the canonical class of $X$ : we obtain $\mathcal{K}_{X}=[-4,1,-106] \in \mathbb{Z}^{3}$.

$>\operatorname{fujitaBPF}(\mathrm{X},[-4,1,-106])$;

To obtain this result the algorithm performs the following steps:

- First Algorithm 2.8 is used to compute the three generators of $[0,0,1],[0,1,0]$ and $[1,0,49]$ of $\operatorname{BPF}(X) \subseteq \mathbb{Z}^{3}$.

- Then Algorithm 2.12 computes the point $C:=[0,0,0] \in c(\tilde{S} / S)$.

- The faces of cone $(S)$ are given by $F_{1}:=\operatorname{cone}([0,1,0],[1,0,49]), F_{2}:=$ cone $([0,0,1],[1,0,49]), F_{3}:=\operatorname{cone}([0,1,0],[0,0,1])$. The algorithms then computes $\alpha_{1}, \alpha_{2}, \alpha_{3}$ such that $-\mathcal{K}_{X}+C=[4,-1,106]$ defines a point in $\tau_{i}^{\alpha_{i}}$. We obtain $\alpha_{1}=-90, \alpha_{2}=-1$ and $\alpha_{3}=4$ as well as $\nu=4$. Note that $\alpha_{3}=4$ is just the first coordinate of $-\mathcal{K}_{X}+C$.

- Since $\operatorname{dim}(X)+1=7>4=\nu-1$ holds, the algorithm returns true.

For a geometric description of $X$, note that in the language of [7, $X$ admits three elementary contractions two of which are birational small. The other one is a birational divisorial contraction $X \rightarrow Y$ contracting the divisor corresponding to the variable $T_{8}$ of $\operatorname{Cox}(X)$. The variety $Y$ is a smooth intrinsic quadric with generator degrees, relation and semiample cone given by

$$
Q=\left[\begin{array}{cc|cc|c||cccc}
60 & 0 & 48 & 12 & 30 & 1 & 1 & 1 & 1 \\
1 & 1 & 1 & 1 & 1 & 0 & 0 & 0 & 0
\end{array}\right], \quad g=T_{1} T_{2}+T_{3} T_{4}+T_{5}^{2}
$$

and $\operatorname{SAmple}(X)=\operatorname{cone}((1,0),(60,1))$. The center of $\varphi$ is the intersection of $Y$ and the toric prime divisors corresponding to the variables $T_{8}, T_{9} \in \operatorname{Cox}(Y)$. Note that $Y$ allows a closed embedding into the the projectivized split vector bundle

$$
\mathbb{P}\left(\mathcal{O}_{\mathbb{P}_{3}} \oplus \mathcal{O}_{\mathbb{P}_{3}}(12) \oplus \mathcal{O}_{\mathbb{P}_{3}}(30) \oplus \mathcal{O}_{\mathbb{P}_{3}}(48) \oplus \mathcal{O}_{\mathbb{P}_{3}}(60)\right) .
$$

Example 4.7. Here we give an example of a locally factorial variety with a torus action of complexity one that does not fulfill Fujita's base point free conjecture. Note that this represents a difference to the toric case, where Fujino [14 presented a proof of Fujita's base point free conjecture for toric varieties with arbitrary singularities.

$$
\begin{aligned}
& >Q:=\operatorname{matrix}([[0,0,1,0,0,1,1,0,1],[1,1,0,1,1,0,1,1,2]]) ; \\
& Q=\left[\begin{array}{lllllllll}
0 & 0 & 1 & 0 & 0 & 1 & 1 & 0 & 1 \\
1 & 1 & 0 & 1 & 1 & 0 & 1 & 1 & 2
\end{array}\right] \\
& >\mathrm{RL}:=[\mathrm{T}[1] * \mathrm{~T}[2] \wedge 7 * \mathrm{~T}[3] \wedge 8+\mathrm{T}[4] * \mathrm{~T}[5] \wedge 7 * \mathrm{~T}[6] \wedge 8+\mathrm{T}[7] \wedge 8] \text {; } \\
& R L:=\left[T_{1} T_{2}^{7} T_{3}^{8}+T_{4} T_{5}^{7} T_{6}^{8}+T_{7}^{8}\right] \\
& >\mathrm{R}:=\operatorname{createGR}(\mathrm{RL}, \operatorname{vars}(9),[\mathrm{Q}]) \text {; } \\
& R:=G R(9,1,[2,[]]) \\
& >\mathrm{X}:=\operatorname{createMDS}(\mathrm{R},[1,3]) \\
& X:=\operatorname{MDS}(9,1,6,[2,[]])
\end{aligned}
$$




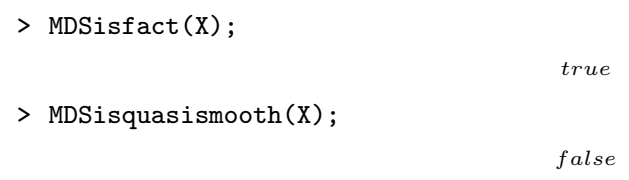

Since $\operatorname{Cox}(X)$ is a complete intersection, we may use the formula presented in [2] to compute the canonical class of $X$ : we obtain $\mathcal{K}_{X}=[4,0] \in \mathbb{Z}^{2}$.

$>\operatorname{fujitaBPF}(\mathrm{X},[4,0])$;

$>$ isBasePointFree $(\mathrm{X},[1,3])$;

$$
\text { false }
$$$$
\text { true }
$$

Note that Algorithm 4.5 returns false, i.e. $X$ does not fulfill Fujita's base point free conjecture. To obtain this result the algorithm performs the following steps:

- First Algorithm 2.8 is used to compute the generators $[0,1]$ and $[1,2]$ of $\operatorname{BPF}(X) \subseteq \mathbb{Z}^{3}$.

- Then Algorithm 2.12 computes the point $C:=[0,0] \in c(\tilde{S} / S)$.

- The faces of cone $(S)$ are given by $F_{1}:=\operatorname{cone}([1,2]), F_{2}:=\operatorname{cone}([0,1])$. The algorithms then computes $\alpha_{1}, \alpha_{2}$ such that $-\mathcal{K}_{X}+C=[-4,0]$ defines a point in $\tau_{i}^{\alpha_{i}}$. We obtain $\alpha_{1}=8, \alpha_{2}=-4$ and $\nu=8$. Note that $\alpha_{2}=-4$ is just the first coordinate of $-\mathcal{K}_{X}+C$.

- Then the algorithm performs the following steps:

- Since we have $\operatorname{dim}(X)+1=7 \leq m \leq 7=\nu-1$, the algorithm only needs to test the case $m=7$.

* For $i=1$ we have $\left\lfloor\frac{\alpha_{1}-1}{7}\right\rfloor=1$, i.e. only the case $k=1$ needs to be considered. The algorithm yields $G p_{i}^{k}=\{[1,3]\}$.

* Now Algorithm 2.6 is used to test whether $\mathcal{K}_{X}+m G p_{i}^{k} \subseteq S$ holds. We have $\mathcal{K}_{X}+7[1,3]=[11,21]$ which is not contained in cone $(S)$. Thus Algorithm 2.6 returns false.

- Hence the algorithm fujitaBPF returns false.

Note that the $\mathcal{K}_{X}+7[1,3]=[11,21]$ is not semiample and thus not nef. Maeda proved in [23, Proposition 2.1] that $\mathcal{K}_{X}+m \mathcal{L}$ is nef for all $m \geq \operatorname{dim}(X)+1$ and for all $\mathcal{L} \in \operatorname{Ample}(X) \cap \operatorname{Pic}(X)$ if $X$ is an irreducible normal projective variety with at most log terminal singularities. Nevertheless, this example does not contradict the result of Maeda since $X$ is not log terminal: To see this, one can look at the relevant face $\gamma_{134}$ and the corresponding affine variety $X_{\gamma_{134}}:=p_{X}\left(\bar{X}_{\gamma_{134}}\right)$, where $p_{X}: \bar{X}^{\mathrm{ss}}(u) \rightarrow X, u:=[1,3]$, denotes the good quotient with respect to the $\operatorname{Spec}(\mathbb{K}[\mathrm{Cl}(X)])$-action on $\bar{X}:=\operatorname{Spec}(\operatorname{Cox}(X))$ and where we set $\bar{X}_{\gamma_{134}}:=\bar{X}_{T_{1} T_{3} T_{4}}$ as in [2, Construction 3.2.1.3.]. By [1], $X_{\gamma_{134}}$ is log terminal only if the exponents of different monomials are platonic triples. Since this is not the case, we conclude that $X$ is not log terminal.

Observe that the base point free monoid $\operatorname{BPF}(X) \subseteq \mathbb{Z}^{2}$ is saturated and thus the ample class $[1,3]$ is base point free. Although $\mathcal{K}_{X}+7[1,3]=[11,21]$ is not base point free on $X$, a result of [21] implies that $\mathcal{K}_{X}+7[1,3]=[11,21]$ is very ample and thus base point free on $X^{\text {reg }}$.

For a geometric description of $X$, note that $X$ admits an elementary contraction $\varphi: X \rightarrow \mathbb{P}_{4}$ of fiber type in the sense of [7] with fibers isomorphic to a hypersurface of degree eight in $\mathbb{P}_{3}$. To be precise we have $\varphi^{-1}(a) \cong V_{\mathbb{P}_{3}}\left(a_{1} a_{2}^{7} T_{0}^{8}+a_{3} a_{4}^{7} T_{1}^{8}+T_{2}^{8}\right)$ where $a=\left[a_{1}, \ldots, a_{5}\right] \in \mathbb{P}_{4}$ denotes a point of $\mathbb{P}_{4}$ in homogeneous coordinates and where $T_{0}, T_{1}, T_{2}, T_{3}$ denote the coordinates of $\operatorname{Cox}\left(\mathbb{P}_{3}\right)$. In addition, $X$ admits a closed embedding $X \rightarrow Y$ into the projectivized split vector bundle

$$
Y=\mathbb{P}\left(\mathcal{O}_{\mathbb{P}_{4}} \oplus \mathcal{O}_{\mathbb{P}_{4}} \oplus \mathcal{O}_{\mathbb{P}_{4}}(1) \oplus \mathcal{O}_{\mathbb{P}_{4}}(2)\right) .
$$

We now turn to the proof of Algorithm 4.5 
Lemma 4.8. In the setting of 4.4, the following are equivalent:

(i) $\mathcal{K}_{X}+m \mathcal{L} \in S$ holds for all $m \geq \operatorname{dim}(X)+1$ and for all ample Cartier divisor classes $\mathcal{L}$, i.e. $X$ fulfills Fujita's base point free conjecture.

(ii) $\mathcal{K}_{X}+m \mathcal{L} \in S$ holds for all $\nu-1 \geq m \geq \operatorname{dim}(X)+1$ and for all ample Cartier divisor classes $\mathcal{L}$.

Proof. Only implication "(ii) $\Rightarrow(\mathrm{i})$ " needs to be proven. Let $m \geq \operatorname{dim}(X)+1$. If $m \leq \nu-1$ holds, then $\mathcal{K}_{X}+m \mathcal{L} \in S$ follows by (ii). Now assume that $m \geq \nu$ holds. Note that since $\mathcal{L}$ defines a point in the relative interior of $\sigma$ for all $1 \leq i \leq r$, the multiple $m \mathcal{L}^{0} \otimes 1$ is contained in a facet parallel $\tau_{i}^{\beta_{i}}$ with $\beta_{i} \geq m \geq \nu$. Thus by definition of $\nu$ as maximum over all integers $\alpha_{i}$ with $\left(-\mathcal{K}_{X}^{0}+C^{0}\right) \otimes 1 \in \tau_{i}^{\alpha_{i}}$, we obtain

$$
m \mathcal{L} \otimes 1 \in\left(\left(-\mathcal{K}_{X}+C\right) \otimes 1\right)+\operatorname{cone}(S) .
$$

Thus, $\mathcal{K}_{X}+m \mathcal{L}$ defines a point in $(C \otimes 1)+\operatorname{cone}(S)$. Since $C$ is an element of the conductor ideal $c(\tilde{S} / S)$ of $S \subseteq K$, we conclude $\mathcal{K}_{X}+m \mathcal{L} \in S$.

Lemma 4.9. In the setting of 4.4, the following are equivalent for $m \in\{\operatorname{dim}(X)+$ $1, \ldots, \nu-1\}:$

(i) $\mathcal{K}_{X}+m \mathcal{L} \in S$ holds for all ample Cartier divisor classes $\mathcal{L}$.

(ii) For all $1 \leq i \leq r$ and for all $1 \leq k \leq\left\lfloor\frac{\alpha_{i}-1}{m}\right\rfloor$, where $\lfloor\cdot\rfloor$ denotes the floor function, we have $\mathcal{K}_{X}+m \mathcal{L} \in S$ for all $\mathcal{L} \in \iota_{0}^{-1}\left(\tau_{i}^{k} \cap \sigma^{\circ}\right) \times K^{\text {tor }}$.

Proof. Only implication "(ii) $\Rightarrow$ (i)" needs to be proven. Consider an ample Cartier divisor class $\mathcal{L}$, i.e.

$$
\mathcal{L} \in \iota_{0}^{-1}\left(\sigma^{\circ}\right) \times K^{\text {tor }}
$$

holds. Let $\beta_{1}, \ldots, \beta_{r} \in \mathbb{Z}_{>0}$ such that $\mathcal{L}^{0} \otimes 1 \in \tau_{i}^{\beta_{i}}$ holds. If $\beta_{i} \leq\left\lfloor\frac{\alpha_{i}-1}{m}\right\rfloor$ holds for some $1 \leq i \leq r$, then $\mathcal{K}_{X}+m \mathcal{L} \in S$ follows by (ii). Now assume that $\beta_{i}>\left\lfloor\frac{\alpha_{i}-1}{m}\right\rfloor$ holds for all $1 \leq i \leq r$. We obtain $m \beta_{i} \geq \alpha_{i}$ for all $1 \leq i \leq r$. Recall that $\left(-\mathcal{K}_{X}^{0}+C^{0}\right) \otimes 1 \in \tau_{i}^{\alpha_{i}}$ holds for all $1 \leq i \leq r$. Thus $m \beta_{i} \geq \alpha_{i}$ for all $1 \leq i \leq r$ shows that

$$
m \mathcal{L} \otimes 1 \in\left(\left(-\mathcal{K}_{X}+C\right) \otimes 1\right)+\operatorname{cone}(S)
$$

holds. Thus, $\mathcal{K}_{X}+m \mathcal{L}$ defines a point in $(C \otimes 1)+\operatorname{cone}(S)$. Since $C$ is an element of the conductor ideal $c(\tilde{S} / S)$ of $S \subseteq K$, we conclude $\mathcal{K}_{X}+m \mathcal{L} \in S$.

Lemma 4.10. Recall that in the setting of 4.4 we defined by $m_{1}, \ldots, m_{n_{i}} \in S$ those elements such that $m_{j}^{0}$ is minimal with the property that $m_{j}^{0} \otimes 1$ is contained in a ray of $F_{i}$. Let $1 \leq i \leq r, 1 \leq k \leq\left\lfloor\frac{\alpha_{i}-1}{m}\right\rfloor$ and consider an ample Cartier divisor class $\mathcal{L} \in \iota_{0}^{-1}\left(\tau_{i}^{k} \cap \sigma^{\circ}\right) \times K^{\mathrm{tor}}$. Then there are $y \in G p_{i}^{k}$ and $a_{j} \in \mathbb{Z}_{\geq 0}$ such that we have

$$
\mathcal{L}=y+\sum_{j=1}^{n_{i}} a_{j} m_{j} .
$$

Proof. Observe that $\sigma \cap \tau_{i}^{k}=\operatorname{conv}\left(p_{1}^{k}, \ldots, p_{t_{i}}^{k}\right)+\operatorname{cone}\left(G_{i}\right)$ holds. Hence there are rational numbers $a_{j}, b_{\ell} \in \mathbb{Q}_{\geq 0}, \sum_{j=1}^{t_{i}} a_{j}=1$, such that the free part of $\mathcal{L}$ is given by

$$
\mathcal{L}^{0}=\sum_{j=1}^{t_{i}} a_{j} p_{j}^{k}+\sum_{\ell=1}^{n_{i}} b_{\ell} m_{\ell}^{0}
$$

We obtain $\mathcal{L}=y+\sum_{\ell=1}^{n_{i}}\left\lfloor b_{\ell}\right\rfloor m_{\ell}(4.101)$, where $\lfloor\cdot\rfloor$ denotes the floor function and where the free and the torsion part of $y$ are defined as

$$
y^{0}:=\sum_{j=1}^{t_{i}} a_{j} p_{j}^{k}+\sum_{\ell=1}^{n_{i}}\left(b_{\ell}-\left\lfloor b_{\ell}\right\rfloor\right) m_{\ell}^{0}, \quad y^{\text {tor }}:=\mathcal{L}^{\text {tor }}-\sum_{\ell=1}^{n_{i}}\left\lfloor b_{\ell}\right\rfloor m_{\ell}^{\text {tor }} .
$$


Note that $y$ is an element of $K$ since we have $y=\mathcal{L}-\sum_{\ell=1}^{n_{i}}\left\lfloor b_{\ell}\right\rfloor m_{\ell}$, where $\mathcal{L}$ as well as the $m_{\ell}, 1 \leq \ell \leq n_{i}$, are elements of $K$. If $y^{0} \otimes 1 \in \sigma^{\circ}$ holds, (4.10,1) is the required representation of $\mathcal{L}$. Now consider the case where $y^{0} \otimes 1$ is not contained in $\sigma^{\circ}$. This means that $y^{0} \otimes 1 \in\left(\operatorname{conv}\left(p_{1}^{k}, \ldots, p_{t_{i}}^{k}\right) \backslash \sigma^{\circ}\right)$ holds. Since $\mathcal{L}^{0} \otimes 1$ is contained in $\sigma^{\circ}$, there is $1 \leq \ell \leq n_{i}$ with $\left\lfloor b_{\ell}\right\rfloor \neq 0$. Without loss of generality we assume that $\left\lfloor b_{1}\right\rfloor, \ldots,\left\lfloor b_{\ell_{0}}\right\rfloor>0$ and $\left\lfloor b_{\ell_{0}+1}\right\rfloor=\ldots=\left\lfloor b_{\ell_{n_{i}}}\right\rfloor=0$ hold for some $1 \leq \ell_{0} \leq n_{i}$. Then we have

$$
\mathcal{L}=y^{\prime}+\sum_{j=1}^{\ell_{0}}\left(\left\lfloor b_{j}\right\rfloor-1\right) m_{j}[4.10,2), \quad \text { where } \quad y^{\prime}:=y+\sum_{j=1}^{\ell_{0}} m_{j}
$$

holds. In order to show that formula [4.10,2) is the required representation of $\mathcal{L}$, it remains to prove that $y^{\prime} \in G_{p_{i}}^{k}$ holds. Note that $y^{\prime} \in K$ holds since $y$ is an element of $K$. In addition, since $y^{0} \otimes 1 \in\left(\operatorname{conv}\left(p_{1}^{k}, \ldots, p_{t_{i}}^{k}\right) \backslash \sigma^{\circ}\right)$ holds, $y^{\prime}$ defines a point in $\operatorname{conv}\left(p_{1}^{k}, \ldots, p_{t_{i}}^{k}\right)+G_{i}$. It remains to show that $y^{\prime}$ defines a point in the relative interior of $\sigma$. Recall that $\sum_{j=1}^{\ell} m_{j}^{0} \otimes 1$ is contained in the facet $F_{i}$. Furthermore, since we are in the case $y^{0} \otimes 1 \notin \sigma^{\circ}$, the point $y^{0} \otimes 1$ lies in a facet $F_{y}$ of $\sigma$. Since $k \geq 1$ and $y \in \iota_{0}^{-1}\left(\tau_{i}^{k}\right)$ hold, we conclude that $y^{0} \otimes 1$ is not contained in $F_{i}$, i.e. there is no face $\kappa \preceq \sigma$ with $y^{0} \otimes 1 \in \kappa$ and $\sum_{j=1}^{\ell} m_{j}^{0} \otimes 1 \in \kappa$. Thus the sum $y^{0}+\sum_{j=1}^{\ell} m_{j}^{0}$ defines a point in the relative interior of $\sigma$. As argued above, this shows that $y^{\prime}$ is an element of $G_{p_{i}}^{k}$, which completes the proof.

Lemma 4.11. In the setting of 4.4. let $\operatorname{dim}(X)+1 \leq m \leq \nu-1, \quad 1 \leq i \leq r$ and $1 \leq k \leq\left\lfloor\frac{\alpha_{i}-1}{m}\right\rfloor$. Then the following are equivalent:

(i) $\mathcal{K}_{X}+m \mathcal{L} \in S$ holds for all $\mathcal{L} \in \iota_{0}^{-1}\left(\tau_{i}^{k} \cap \sigma^{\circ}\right) \times K^{\text {tor }}$.

(ii) $\mathcal{K}_{X}+m \mathcal{L} \in S$ holds for all $\mathcal{L} \in G p_{i}^{k}$.

Proof. Since $G p_{i}^{k} \subseteq \iota_{0}^{-1}\left(\tau_{i}^{k} \cap \sigma^{\circ}\right) \times K^{\text {tor }}$ holds, only implication "(ii) $\Rightarrow$ (i)" needs to be proven. Note that this is an immediate consequence of Lemma 4.10.

Proof of Algorithm 4.5. We need to show that $X$ fulfills Fujita's base point free conjecture if and only if the above algorithm returns true. This can be seen as follows: if $X$ is not Gorenstein, then $\mathcal{K}_{X}+m \mathcal{L}$ is not a Cartier divisor class; in particular, it is not base point free. Now assume that $X$ is Gorenstein. Since the embedded monoid $\operatorname{BPF}(X) \subseteq \operatorname{Pic}(X)$ is spanning, we can apply Algorithm 2.12 and compute a point of its conductor ideal. Lemma 4.8 shows that we can bound $m$ by $\nu-1$; Lemmata 4.9 and 4.11 prove that the sets $G p_{i}^{k}, 1 \leq i \leq r, 1 \leq k \leq\left\lfloor\frac{\alpha_{i}-1}{m}\right\rfloor$, serve as validations sets of Cartier divisor classes.

\section{REFERENCES}

[1] I. Arzhantsev, L. Braun, J. Hausen, M. Wrobel: Log terminal singularities, platonic tuples and iteration of Cox rings. European Journal of Mathematics, to appear.

[2] I. Arzhantsev, U. Derenthal, J. Hausen, A. Laface: Cox rings. Cambridge Studies in Advanced Mathematics no. 144, Cambridge Univ. Press, Cambridge (2014).

[3] C. Birkar, P. Cascini, C. Hacon, J. McKernan: Existence of minimal models for varieties of log general type. J. Amer. Math. Soc. 23 (2010), 405-468.

[4] M. Brion: Curves and divisors in spherical varieties. In Algebraic groups and Lie groups, Austral. Math. Soc. Lect. Ser., vol. 9, Cambridge Univ. Press, Cambridge (1997), 21-34

[5] M. Brion, F. Knop: Contractions and flips for varieties with group action of small complexity.

J. Math. Sci. Univ. Tokyo vol. 1 (1994), no. 3, 641-655.

[6] W. Bruns, J. Gubeladze: Polytopes, Rings, and K-Theory. Springer, Heidelberg (2009).

[7] C. Casagrande: On the birational geometry of Fano 4-folds. Mathematische Annalen (2013), no. 355, 585-628.

[8] D.A. Cox, J.B. Little, H.L. Schenck: Toric varieties. Graduate Studies in Mathematics, 124. American Mathematical Society, Providence, RI, 2011.

[9] O. Debarre: Higher-dimensional algebraic geometry. Universitext, Springer-Verlag, New York, 2001. 
[10] L. Ein, R. Lazarsfeld: Global generation of pluricanonical and adjoint linear series on smooth projective threefolds. J. Amer. Math. Soc. vol. 6 no. 4 (1993), 875-903.

[11] M. Franz: Convex a Maple package for convex geometry. Available at http://www.math.uwo.ca/ mfranz/convex/

[12] A. Fahrner: Monoid Package - a package for monoids and base point free problems of Mori dream spaces. Available at http://www.math.uni-tuebingen.de/user/fahrner/MonoidPackage/

[13] A. Fahrner, J. Hausen, M. Nicolussi: Smooth projective varieties with a torus action of complexity 1 and Picard number 2. To appear in Annali della Scuola Normale Superiore di Pisa.

[14] O. Fujino: Notes on toric varieties from Mori theoretic viewpoint. Tohoku Math. J. (2) vol. 55 no. 4 (2003), 551-564.

[15] T. Fujita: Problems. In: Birational Geometry of Algebraic Varieties: Open Problems, Katata Syposium of the Taniguchi Foundation (1988), 42-47.

[16] J. Hausen, E. Herppich, H. Süß: Multigraded factorial rings and Fano varieties with torus action. Documenta Math. 16 (2011), 71-109.

[17] J. Hausen, S. Keicher: A software package for Mori dream spaces. LMS J. Comput. Math. vol. 18 no. 1 (2015), 647-659.

[18] Y. Hu, S. Keel: Mori dream spaces and GIT. Dedicated to William Fulton on the occasion of his 60th birthday. Michigan Math. J. 48 (2000), 331-348.

[19] N.O. Ilten, R. Vollmert: Upgrading and Downgrading Torus Actions. J. Pure Appl. Algebra 217 (2013) no. 9, 1583-1604.

[20] Y. Kawamata: On Fujita's freeness conjecture for 3-folds and 4-folds. Math. Ann. vol. 308 no. 3 (1997), 491-505.

[21] A. Lanteri, M. Palleschi, A. J. Sommese: Very ampleness of $K_{X} \otimes \mathcal{L}^{\operatorname{dim}(X)}$ for ample and spanned line bundles $\mathcal{L}$. Osaka J. Math. 26 (1989), no. 3, 647-664.

[22] D. Luna: Grosses cellules pour les variétés sphériques. In Algebraic Groups and Lie Groups, ed. by G. I. Lehrer, Australian Math. Soc. Lecture, Series 9 (1997), 267-280.

[23] H. Maeda: Ramification divisors for branched coverings of $\mathbb{P}_{n}$. Math. Ann. 288 (1990), no. 2, 195-199.

[24] J. McKernan: Mori dream spaces. Jpn. J. Math. 5 (2010), no. 1, 127-151.

[25] L. Petersen, H. Sü B: Torus invariant divisors. Israel Journal of Mathematics, vol. 182, 2011, p.481-505.

[26] I. Reider: Vector bundles of rank 2 and linear systems on algebraic surfaces. Ann. of Math. 2 (1988), vol. 127 no.2, 309-316.

[27] F. Ye, Z. Zhu: On Fujita's freeness conjecture in dimension 5. ArXiv e-prints 2015.

Mathematisches Institut, Universität Tübingen, Auf Der Morgenstelle 10, 72076 TüBingen, Germany

E-mail address: fahrner@math.uni-tuebingen.de 